\title{
Role of Ephemerals in Sustainability of Grazing Lands in Arid Areas
}

\author{
Suresh Kumar* \\ Central Arid Zone Research Institute, India
}

Received: 钢 September 06, 2018; Published: 眥 September 17, 2018

*Corresponding author: Suresh Kumar, Central Arid Zone Research Institute, Current Address-124, Subhash Nagar, Pal Road, Jodhpur- 342 008, India

\begin{abstract}
Ephemeral species germinate with onset of rains and complete their lifecycle by setting seeds as the soil moisture declines within one growing season. Though their life cycle is short, they play a multifaceted role in ecological and economic sustenance of arid regions in general and grazing lands in particular. This role of ephemerals emanating from various studies has been synthesized here in respect of Indian arid zone. Nearly half of the reported 682 species in the Indian arid zone are ephemeral and seasonal, the majority being monsoonal. They constitute bulk of alpha diversity on any landscape. These occur on all land uses i.e., croplands and grazing lands. Species composition of ephemerals which varies on different habitats and in varying annual precipitation has been described in this paper.

Changes is species composition, cover and biomass of ephemerals due to grazing have been discussed. Comparing phenology of selected seasonals in different grazing pressures with those in protected areas revealed that increasing grazing stress caused a typical shift in their phenophases. Production potential of ephemerals estimated in a large variety of situations indicated that they contribute differently to the total biomass of the grazing lands representing different range conditions. Since ephemerals being palatable offer grazable material, their role in prolonging duration of range use by the livestock has been proved from case studies. Besides, ephemerals occur as weeds of cropland, their composition and biomass on different habitats have been described to assess their contribution to liverstock support. The ecological value of self regenerated ephemerals in rehabilitation of disturbed lands such as mine spoils has been discussed. Many of these ephemerals are also medicinally important with proven economic potential for enhancing the livelihood of desert dwellers.
\end{abstract}

Keywords: Ephemerals; Sustainability; Grazing; Phenophase; Biomass

\section{Introduction}

Arid regions experience a spurt in vegetation emergence during monsoon. While perennials regenerate, the annual species germinate to put up a sprawling green cover bringing profound changes in the desert landscape. These include changes in species compositions, phenology, production and utilization of these ephemerals vis-a-vis perennials. While extensive literature exists on these aspects in respect of perennials, little attention seems to have been paid to the desert ephemerals. Secondly, twelve extreme arid districts in western Rajasthan have $67.2 \%$ area under culturable waste which is used as grazing ground [1]. This region provides highest quantum of meat, milk, and wool to the country from an area (36\%) of which $2 / 3$ area is largely a degraded wasteland.
Encroachment of these grazing lands is shrinking their area on one hand while quality of the feed is also declining due to overstocking. How constantly shrinking grazing lands in this region, which are also declining in feed quality with less perennial grasses sustain enhancing livestock pressure has been a paradox. Do ephemerals play any role in sustainability of these grazing lands was a question that needed an independent investigation for arriving at unbiased conclusion. An attempt has therefore been made in present paper to collate and synthesize results of studies on various such aspects to prove that ephemerals do play an important role in sustainability of grazing lands in arid areas with special reference to Indian arid zone 


\section{The Environment}

Region facing annual water deficit of two - third or more of the potential evapotranspiration (PET) is classified as arid [2]. Semi arid regions experience this deficit ranging from one third to two third of PET. Using this criteria 9.56 lakh $\mathrm{km} 2$ (30.50\%) can be classified as semi arid and 3.81 lakh km2 (10.16\%) area as arid in India. Arid region of India has over $60 \%$ area in western Rajasthan and experience extremes of temperature $\left(0\right.$ to $4{ }^{\circ} \mathrm{C}$ in winter and 45 $48{ }^{\circ} \mathrm{C}$ in summer), low annual precipitation ranging from $450 \mathrm{~mm}$ in the east to $10 \mathrm{~mm}$ in the west, low humidity, high wind velocity and high evapotranspiration. Soils are sandy, poor in nutrient with low water holding capacity and prone to erosion by wind and water. Natural vegetation in such edapho climatic conditions is sparse and stunted, predominantly spiny belonging mainly to grass cover type Dichanthium- Cenchrus-Lasiurus- type and very small area having Sehima- Dichanthium type [3].

\section{Dichanthium - Cenchrus_Lasiurus Cover Type}

This grass cover occurs in the region receiving $100-750 \mathrm{~mm}$ rainfall with higher mean temperature during summer $\left(42-48^{\circ} \mathrm{C}\right)$ and winter temperature as low as 1 to $2{ }^{\circ} \mathrm{C}$ ). These grasses occur mainly on alluvial soils with varying amount of loam having high soluble salts and pale gray and brown colours. The geographical extent of $4.36 \times 10^{5} \mathrm{sq} \mathrm{km}$ area is distributed in northern portion of Gujarat, Rajasthan (excluding Aravallis), western Uttar Pradesh, Delhi State and semi arid Punjab and Haryana [4]. This cover type has 11 perennial grasses, 45 other herbaceous species of which 19 are legumes. The predominant woody perennials here include Acacia senegal, Calotropis procera and Cassia auriculata. These areas look typical as savanna lands.

\section{Floristics of Herbage}

Table 1: Herbage species and their families in the Indian arid zone ( $\mathrm{S}=$ season, $\mathrm{W}=$ winter, $\mathrm{M}=$ monsoon, $\mathrm{A}=$ all $\mathrm{yr}$ ).

\begin{tabular}{|c|c|c|}
\hline Family & Name & S \\
\hline \multirow{2}{*}{ 1. Fumariaceae } & 1. Fumaria Indica & $W$ \\
\hline \multirow{2}{*}{ 3. Brassicaceae } & 2. Farsetia hamiltonii & $M$ \\
\cline { 2 - 3 } & 3. Sisymbrium irio & $W$ \\
\hline \multirow{3}{*}{ 4. Polygalaceae } & 4. Cleome gracilis & $M$ \\
\cline { 2 - 3 } & 5. C. gyanandra & $M$ \\
\hline \multirow{3}{*}{ 5. Caryophyllaceae } & 6. C. vahliana & $A$ \\
\hline & 7. C. viscosa & $M$ \\
\hline & 8. P. erioptera & $M$ \\
\hline & 10. Polygala irregularis & $M$ \\
\hline & 11. Spergula arvensis & $W$ \\
\hline
\end{tabular}

\begin{tabular}{|c|c|c|}
\hline \multirow{5}{*}{ 6. Portulaceae } & 13. Portulaca oleracea & $M$ \\
\hline & 14. P. tuberosa & $M$ \\
\hline & 15. P. quadrifida & M \\
\hline & 16. P. meridiana & M \\
\hline & 17. Talinum rotulacifolium & M \\
\hline \multirow{3}{*}{ 7. Malvaceae } & 18. Hibiscus $s p$. & M \\
\hline & 19. Malva parviflora & $W$ \\
\hline & 20. Malvastrum coromandelianum & $A$ \\
\hline \multirow{7}{*}{ 8. Tiliaceae } & 21. Corchorus aestuans & $M$ \\
\hline & 22. C. depressus & $A$ \\
\hline & 23.C. fascicularis & $M$ \\
\hline & 24. C. olitorius & $M$ \\
\hline & 25. C. tridens & $M$ \\
\hline & 26. C. trilocularis & $M$ \\
\hline & 27. Triumfetta pentandra & $M$ \\
\hline \multirow{6}{*}{ 9. Zygophyllaceae } & 28. Seetzenia lanata & M \\
\hline & 29. Tribulus rajasthanensis & $M$ \\
\hline & 30. T. terrestris & M \\
\hline & 31. T. lanuginosus & $A$ \\
\hline & 32. T. pentendrus & $M$ \\
\hline & 33. Zygophyllum simplex & M \\
\hline 10. Oxalidaceae & 34. Oxalis corniculata & $W$ \\
\hline \multirow{2}{*}{ 11. Sapindaceae } & 35. Cardiospermum halicacabum & $M$ \\
\hline & 36. Alysicorpus glumaceous & $M$ \\
\hline \multirow{24}{*}{ 12. Fabaceae } & 37. A. heterophyllus & $M$ \\
\hline & 38. A. longifolius & $M$ \\
\hline & 39. A. monilifer & $M$ \\
\hline & 40. A. procumbens & $M$ \\
\hline & 41. A. styracifolius & $M$ \\
\hline & 42. A. vaginalis & $M$ \\
\hline & 43. Atylosia scarabaeoides & $M$ \\
\hline & 44. Clitoria ternatea & $M$ \\
\hline & 45. Crotalaria medicaginea & $M$ \\
\hline & 46. Goniogyna hirta & M \\
\hline & 47. Indigofera astragalina & $M$ \\
\hline & 48. I. cordifolia & $M$ \\
\hline & 49. I. hochstetteri & $M$ \\
\hline & 50. I. linifolia & $M$ \\
\hline & 51. I. linnaei & $M$ \\
\hline & 52. I. sessiliflora & $M$ \\
\hline & 53. Lathyrus aphaca & $W$ \\
\hline & 54. L. sativus & $W$ \\
\hline & 55. Macrotyloma uniflorum & $M$ \\
\hline & 56. Medicago sativa & $W$ \\
\hline & 57. Meliltus alba & $W$ \\
\hline & 58. Melilotus indica & $W$ \\
\hline & 59. Rhynchosia aurea & $M$ \\
\hline & 60. R. minima & M \\
\hline
\end{tabular}




\begin{tabular}{|c|c|c|}
\hline & 61. Sesbania bispinosa & $M$ \\
\hline & 62. Tephrosia leptostaehya & $M$ \\
\hline & 63. T. strigosa & $M$ \\
\hline & 64. Trigonella corniculata & $W$ \\
\hline & 65. T. hamosa & $W$ \\
\hline & 66. T. monantha & $W$ \\
\hline & 67. T. occulta & $W$ \\
\hline & 68. Vicia sativa & $W$ \\
\hline & 69. Vigna trilobata & $M$ \\
\hline & 70. Zornia gibbosa & $M$ \\
\hline & 71. Cassia occidentalis & $M$ \\
\hline 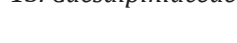 & 72. C. pumila & $M$ \\
\hline & 73. Neurada procumbens & $M$ \\
\hline & 74. Potentilla supina & $W$ \\
\hline & 75. Ammannia baccifera & $M$ \\
\hline 15. Lythraceae & 76. Ammannia desertorum & $M$ \\
\hline & 77. A. multiflora & $M$ \\
\hline 16. Vahliaceae & 78. Bistella digyna & $M$ \\
\hline 17. Onagraceae & 79. Ludwigia perennis & $M$ \\
\hline & 80. Citrullus fistulosus & M \\
\hline & 81. C. lanatus & $M$ \\
\hline & 82. Ctenolepis cerasiformis & $M$ \\
\hline & 83. Cucumis callosus & $M$ \\
\hline & 84. C. melo var agrestis & $M$ \\
\hline 18. Cucurbitaceae & 85. C. melo var momordica & $M$ \\
\hline & 86. C. prophetarum & $M$ \\
\hline & 87. Dactyliandra welwitschii & $M$ \\
\hline & 88. Luffa acutangula & $M$ \\
\hline & 89. Luffa echinata & $M$ \\
\hline & 90. Mukia maderaspatana & $M$ \\
\hline & 91. Corbichonia decumbans & $M$ \\
\hline & 92. Gisekia pharnacioides & $M$ \\
\hline 19. Molluginaceac & 93. Glinus lotoides & $M$ \\
\hline & 94. Mollugo cerviana & $M$ \\
\hline & 95. Mollugo nudicaulis & $M$ \\
\hline & 96. Sesuvium sesuvioides & $M$ \\
\hline$? 0$ - iं & 97. Trianthema potulacastrum & $M$ \\
\hline & 98. T. triquetra & $M$ \\
\hline & 99. Zaleya redimita & $W$ \\
\hline & 100. Borreria articularis & $M$ \\
\hline & 101. Borreria pusila & $M$ \\
\hline 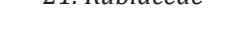 & 102. Hedyotis corymbosa & $M$ \\
\hline & 103. Kohautia aspera & $M$ \\
\hline
\end{tabular}

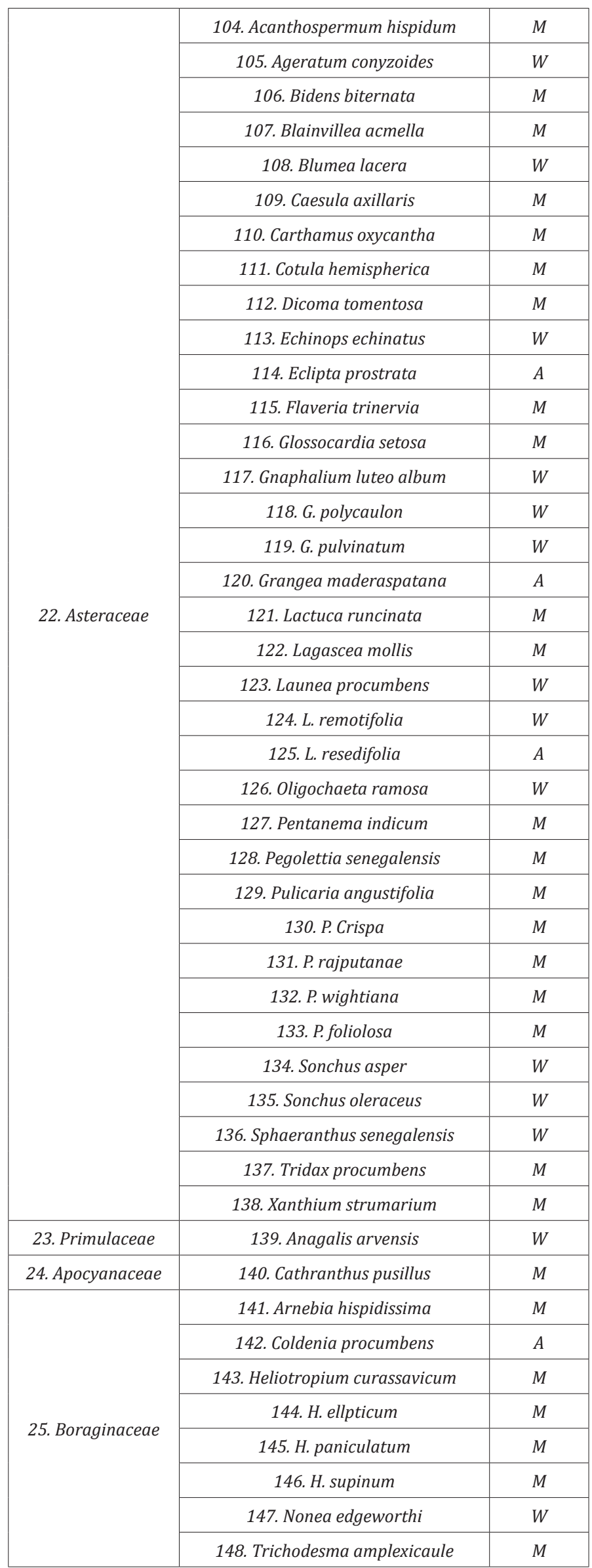




\begin{tabular}{|c|c|c|}
\hline \multirow{14}{*}{ 26. Convolvulaceae } & 149. Convolvulus rhyniospermus & $M$ \\
\hline & 150. Convolvulus stocksii & $M$ \\
\hline & 151. Ipomoea coptica & $M$ \\
\hline & 152. Ipomoea dichroa & $M$ \\
\hline & 153. I. eriocarpa & $M$ \\
\hline & 154. I. hederifolia & $M$ \\
\hline & 155. I. nil & $M$ \\
\hline & 156. I. quamoclit & $M$ \\
\hline & 157. I. sindica & $M$ \\
\hline & 158. I. turbinata & $M$ \\
\hline & 159. I. verticillata & $M$ \\
\hline & 160. Merremia aegyptia & $M$ \\
\hline & 161. M. rajasthanensis & $M$ \\
\hline & 162. Ipomea pestigris & $W$ \\
\hline \multirow{8}{*}{ 27. Solanaceae } & 163. Datura innoxia & $M$ \\
\hline & 164. D. metal & $M$ \\
\hline & 165. D. stramonuim & $M$ \\
\hline & 166. D. ferox & $M$ \\
\hline & 167. Nicotiana plumbaginifiilia & $W$ \\
\hline & 168. Lycoperiscon lycopersicum & $W$ \\
\hline & 169. Physalis minima & $M$ \\
\hline & 170. Solanum nigrum & $M$ \\
\hline \multirow{13}{*}{ 28. Scrophulariacea } & 171. Anticharis glandulosa & $M$ \\
\hline & 172. A. senegalensis & $M$ \\
\hline & 173. Bacopa monnieri & $A$ \\
\hline & 174. Dopatrium junceum & $M$ \\
\hline & 175. Glossostigma diandrum & $W$ \\
\hline & 176. Lindernia parviflora & $M$ \\
\hline & 177. Schweinfurthia papilionacea & $M$ \\
\hline & 178. Sopubia dephinifolia & $M$ \\
\hline & 179. Striga anqustifolia & $M$ \\
\hline & 180. Verbascum chinense & $M$ \\
\hline & 181. Striga gesnerioides & $M$ \\
\hline & 182. Veronica agrestis & W \\
\hline & 183. Veronica anagallis -aquatica & $W$ \\
\hline \multirow{2}{*}{ 29. Orobanchaceae } & 184. Orobanche aegyptica & $W$ \\
\hline & 185. о. cernua & $W$ \\
\hline \multirow{3}{*}{ 30. Pedaliaceae } & 186. Pedalium murex & $M$ \\
\hline & 187. Sesamum indicum & $M$ \\
\hline & 188. S. mulayanum & $M$ \\
\hline 31. Martyniaceae & 189. Proboscidea louisiana & $M$ \\
\hline
\end{tabular}

\begin{tabular}{|c|c|c|}
\hline \multirow{13}{*}{ 32. Acanthaceae } & 190. Blepharis repens & $W$ \\
\hline & 191. B. sindica & $M$ \\
\hline & 192. Dicliptera verticillata & $M$ \\
\hline & 193. Hemiadelphis polyspermus & $W$ \\
\hline & 194. Hygrophylla auriculata & $M$ \\
\hline & 195. Indoneesiella echioides & $M$ \\
\hline & 196. Justicia heterocarpa & $M$ \\
\hline & 197. J. procumbens & $M$ \\
\hline & 198. J. quinquangularis & $M$ \\
\hline & 199. J. simplex & $M$ \\
\hline & 200. Jvahlii & $M$ \\
\hline & 201. Peristrophe paniculata & $M$ \\
\hline & 202. Ruellia tuberosa & $M$ \\
\hline \multirow{6}{*}{ 33. Lamiaceae } & 203. Lavandula bipinnata & $M$ \\
\hline & 204. Leucas aspera & $M$ \\
\hline & 205. Leucas cephalotes & $M$ \\
\hline & 206. L. nutans & $M$ \\
\hline & 207. L. stricta & $M$ \\
\hline & 208. L. urticaefolia & $M$ \\
\hline 34. Plantaginaceae & 209. Plantago exiqua & $W$ \\
\hline \multirow{13}{*}{ 35. Amaranthaceae } & 210. Achyranthes aspera & $M$ \\
\hline & 211. Alternanthera pungens & $A$ \\
\hline & 212. A. sessilis & $M$ \\
\hline & 213. Amaranthus blitum & $M$ \\
\hline & $\begin{array}{l}\text { 214. A. graecizans subsp. } \\
\text { thellungiamus }\end{array}$ & $M$ \\
\hline & 215. A. hybridus subsp cruentus & $M$ \\
\hline & 216. A. spinosus & $M$ \\
\hline & 217. A. tricolor & $M$ \\
\hline & 218. Celosia argentia & \\
\hline & 219. Digera muricata & $M$ \\
\hline & 220. Gomphrena celosioides & $M$ \\
\hline & 221. Nothosaerva brachiata & $A$ \\
\hline & 222. Chenopodium album & $M$ \\
\hline \multirow{2}{*}{36 Chenopodiaceae } & 223. C. murale & $W$ \\
\hline & 224. Emex spinosa & $W$ \\
\hline \multirow{3}{*}{ 37. Polygonaceae } & 225. Polygonum plebium & $W$ \\
\hline & 226. Rumex dentatus & $W$ \\
\hline & 227. Peperomia pellucida & $M$ \\
\hline
\end{tabular}




\begin{tabular}{|c|c|c|}
\hline 38. Piperaceae & 228. Acalypha ciliata & $M$ \\
\hline \multirow{19}{*}{ 39. Euphorbicaceae } & 229. Acalypha ciliata & $M$ \\
\hline & 230. A. indica & $M$ \\
\hline & 231. Chrozophora prostrata & $A$ \\
\hline & 232. C'. rottleri & $W$ \\
\hline & 233. Euphorbia chamaesyce & $A$ \\
\hline & 234. E. clarkeana & $M$ \\
\hline & 235. E. dracunculiodes & M \\
\hline & 236. E. elegans & $M$ \\
\hline & 237. E. geniculata & M \\
\hline & 238. E. granulata & $M$ \\
\hline & 239. E. heyneana & M \\
\hline & 240. E. hirta & $A$ \\
\hline & 241. E. indica & M \\
\hline & 242. E. jodhpurensis & $M$ \\
\hline & 243. E. thymifolia & $M$ \\
\hline & 244. Micrococca mercurialis & M \\
\hline & 245. Phvllanthus amarus & M \\
\hline & 246. P. fraternus & M \\
\hline & 247. P. maderaspatensis & $M$ \\
\hline \multirow{2}{*}{ 40. Liliaceae } & 248. Dipcadi erythraeum & M \\
\hline & 249. Amischophacelus axillaris & $M$ \\
\hline \multirow{5}{*}{ 41. Commelinaceae } & 250. Commelina benghalensis & M \\
\hline & 251. C. diffutsa & $M$ \\
\hline & 252. C. forskalaei & $M$ \\
\hline & 253. Cyanotis cristata & $M$ \\
\hline & 254. Juncus bufonius & $w$ \\
\hline \multirow{20}{*}{ 43. Cyperaceae } & 256. C. compressus & $M$ \\
\hline & 257.C. cuspidatus & $M$ \\
\hline & 258. C. difformis & $M$ \\
\hline & 259. C.flavidus & M \\
\hline & 260. Ciria & M \\
\hline & 261. C. michelianus & $M$ \\
\hline & 262. C. pumilus & $M$ \\
\hline & 263. C. triceps & $M$ \\
\hline & 264. Eleocharis atropurpurea & M \\
\hline & 265. E. geniulata & $M$ \\
\hline & 267. F. bisumbellata & $M$ \\
\hline & 268. F. quinquangularis & $M$ \\
\hline & 269. F. tenera & M \\
\hline & 270. Mariscus squarosus & $M$ \\
\hline & 271. Scirpus grossus & $M$ \\
\hline & 272. S. littoralis & $M$ \\
\hline & 273.S. roylei & $M$ \\
\hline & 274. S. supinus & M \\
\hline & 275. S. tuberosus & $M$ \\
\hline & 276. Andropogon pumilus & M \\
\hline
\end{tabular}

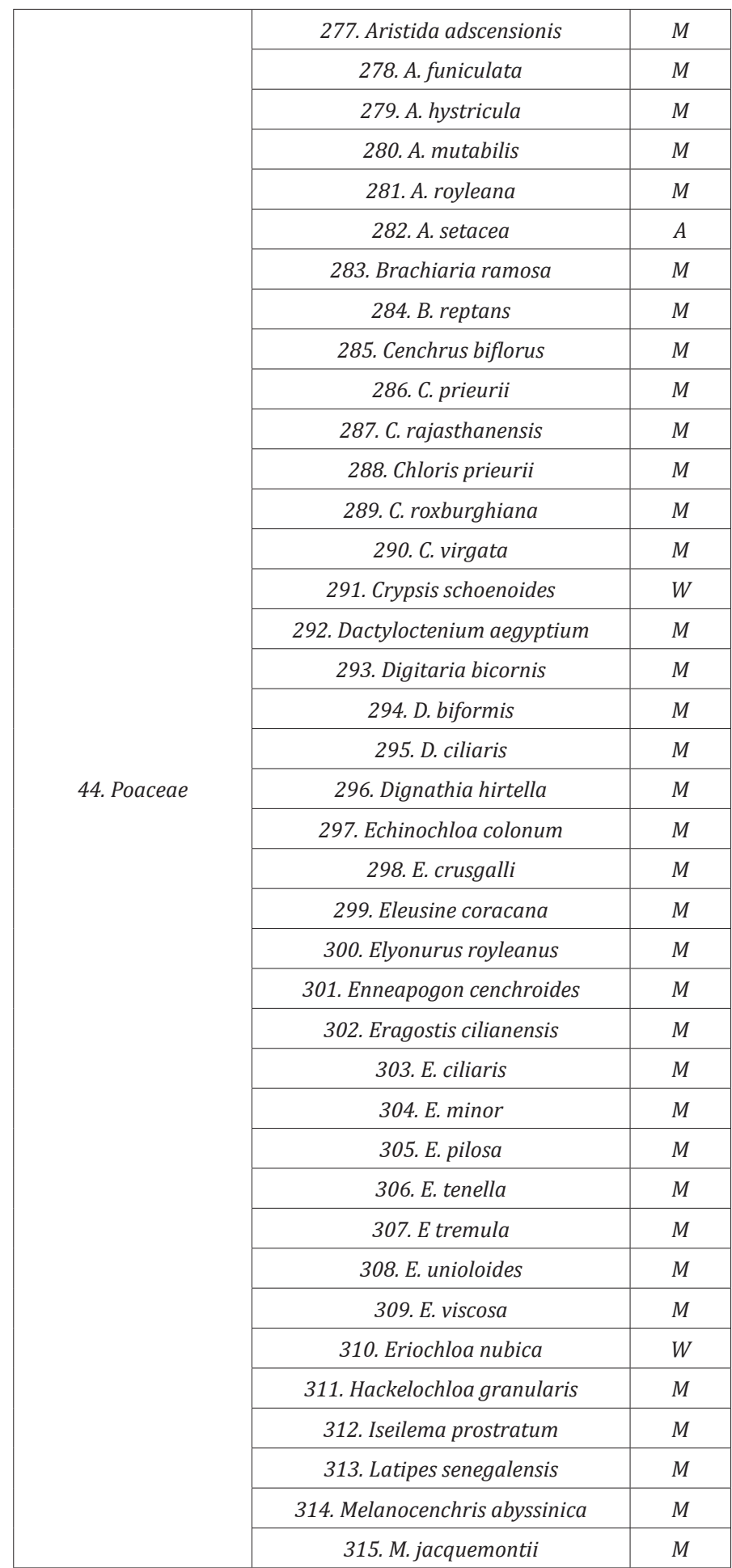




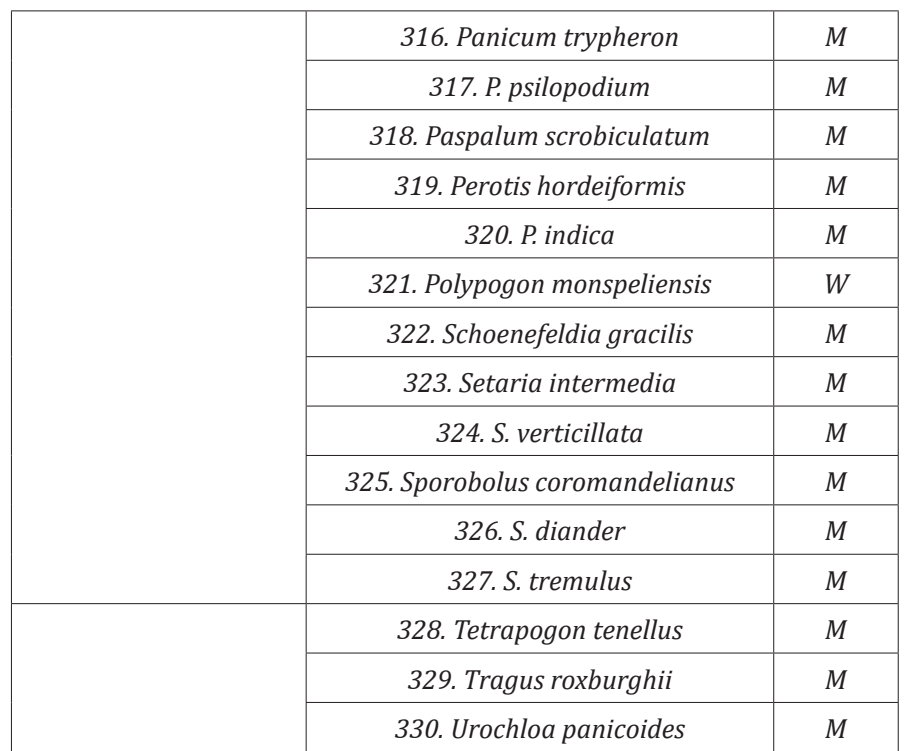

Arid part of Rajasthan has 682 species. Of these 330 are ephemerals belonging to 44 families [5] (Table 1). Hydrophytes have been excluded from this analysis. Some 54 species are winter ephemerals; 17 occur in both monsoon and winter. In true sense, some 260 species (38.12\% of flora) constitute monsoon herbage.
These belong to 188 genera and 44 families. Amongst families, Poaceae has maximum i.e. 55 monsoon species followed by 35 each in Fabaceae and Asteraceae,

\section{Herbage Communties}

Vegetation is a reflex of climate, land form and its surface deposit. Broadly classified on the basis of physiognomy, six characteristic types are recognized in the Indian arid zone [6]. These are

a) Mixed xeromorphic thorn forest on hill and rock outcrops.

b) Mixed xeromorphic wood lands on piedmonts and alluvial plains.

c) Mixed xeromorphic riverine thorn forest on younger alluvial plain around desertic river and water bodies.

d) Lithophytes scrub on eroded rocky gravelly plains.

e) Psammophytic scrub on sand dunes, hummocks and sandy plains.

f) Halophytic scrub on low lying saline flats or ranns (Table 2).

Table 2: Vegetation types of Indian Arid zone.

\begin{tabular}{|c|c|c|c|c|c|}
\hline Parameters & \multicolumn{3}{|c|}{ Mixed xeromorphic Thorn Forests } & $\begin{array}{l}\text { Mixed xeromorphic } \\
\text { wood lands }\end{array}$ & $\begin{array}{l}\text { Mixed xeromorphic } \\
\text { Riverine thorn forests }\end{array}$ \\
\hline Habitats & \multicolumn{3}{|c|}{ Hills of rhyolite, granite, sandstone } & $\begin{array}{l}\text { Pediments order } \\
\text { alluvial plains } \\
\text { underlain with kankar } \\
\text { at } 25-100 \mathrm{~cm} \text { depth }\end{array}$ & $\begin{array}{c}\text { Younger alluvium around } \\
\text { desert rivers }\end{array}$ \\
\hline Soils & \multicolumn{3}{|c|}{ Skeletal, loamy sand } & Sandy loam-clay loam & $\begin{array}{c}\text { Deep, sandy soils without } \\
\text { hard pan }\end{array}$ \\
\hline \multirow{3}{*}{$\begin{array}{l}\text { Community } \\
\text { Physiognomy }\end{array}$} & \multicolumn{3}{|c|}{ Thorny and spiny } & \multirow{3}{*}{$\begin{array}{l}\text { A mix of thorny and } \\
\text { evergreen species }\end{array}$} & \multirow{3}{*}{$\begin{array}{c}\text { Thorny and evergreen } \\
\text { species }\end{array}$} \\
\hline & \multicolumn{3}{|c|}{ Annual precipitation } & & \\
\hline & $150-350 \mathrm{~mm}$ & $350-500 \mathrm{~mm}$ & $500-700 \mathrm{~mm}$ & & \\
\hline Woody perennials & $\begin{array}{c}\text { Acacia senegal } \\
\text { Salvadora oleoides, } \\
\text { Commiphora wightii, } \\
\text { Ziziphus nummularia, } \\
\text { Grewia tenax }\end{array}$ & $\begin{array}{c}\text { Anogeissus pendula, } \\
\text { Acacia senegal, } \\
\text { Wrightia tinctoria, } \\
\text { Moringa concanensis, } \\
\text { Bauhnia racemosa, } \\
\text { Cordia gharaf, } \\
\text { A. leucophloea, } \\
\text { Azadirachta indica }\end{array}$ & $\begin{array}{l}\text { A. pendula, } \\
\text { Acacia catechu, } \\
\text { Boswellia serrata, } \\
\text { Dichrostachys } \\
\text { cinerea, } \\
\text { Grewia tenax, } \\
\text { Dipteracanthus } \\
\text { potulus } \\
\end{array}$ & $\begin{array}{c}\text { Prosopis cineraria, } \\
\text { Salvadora oleoides, } \\
\text { Acacia nilotica, } \\
\text { Capparis decidua, } \\
\text { Ziziphus nummularia, } \\
\text { Calotropis procera, } \\
\text { Balanites aegyptiaca }\end{array}$ & $\begin{array}{c}\text { Acacia nilotica, A. nilotica } \\
\text { sub sps. cupressiformis, } \\
\text { Salvadora oleoides, } \\
\text { Tecomella undulata, } \\
\text { Tamarindus indica, } \\
\text { Tamarix Sps., Acacia } \\
\text { jacquemontii, Cassia } \\
\text { auriculata } \\
\end{array}$ \\
\hline $\begin{array}{l}\text { Herbaceous annuals } \\
\text { and perennials }\end{array}$ & $\begin{array}{c}\text { Aristida hirtigluma, } A \text {. } \\
\text { funiculata, Enneapogon } \\
\text { brachystachys }\end{array}$ & $\begin{array}{r}\text { Tephrosia petrosa, Bra } \\
\text { procumbens, Ach }\end{array}$ & $\begin{array}{l}\text { aria ramosa, Tridex } \\
\text { anthes aspera }\end{array}$ & $\begin{array}{l}\text { Zornia diphylla, } \\
\text { Achyranthes } \\
\text { aspera, Alysicarpus } \\
\text { spp., Commelina } \\
\text { benghalensis, } \\
\text { Eragrostis spp., } \\
\text { Fagonia cretica }\end{array}$ & $\begin{array}{l}\text { Tetrapogon tenellus, } \\
\text { Zornia disphylla, } \\
\text { C. boflorus, Tragus } \\
\text { roxburghii, C. prieurii, } \\
\text { Dactyloctenium } \\
\text { aegyptium. }\end{array}$ \\
\hline Parameters & Lithophytic Scrub & Psammopl & c Scrub & Haloph & tic Scrub \\
\hline Habitats & $\begin{array}{c}\text { Eroded rocky surfaces } \\
\text { gravelly plain, } \\
\text { pediment plain. }\end{array}$ & $\begin{array}{r}\text { Sands dunes, hummo } \\
\text { humm }\end{array}$ & $\begin{array}{l}\text { Sandy plains with } \\
\text { cks }\end{array}$ & Low lying sal & he flats or ranns \\
\hline Soils & $\begin{array}{l}\text { Shallow soil deposition } \\
\text { in pockets }\end{array}$ & $\begin{array}{r}\text { Deep loamy sandy s } \\
\text { deposits ofte }\end{array}$ & $\begin{array}{l}\text { s, mainly aeolian } \\
\text { alcareous }\end{array}$ & Deep s & ne dry soil \\
\hline
\end{tabular}




\begin{tabular}{|c|c|c|c|c|}
\hline \multirow{2}{*}{$\begin{array}{l}\text { Community } \\
\text { physiognomy }\end{array}$} & \multirow{2}{*}{$\begin{array}{l}\text { Stunted, } \\
\text { multistemmed shrubs, } \\
\text { grazed. }\end{array}$} & \multicolumn{2}{|c|}{ Stunted, multistemmed shrubs, grazed. } & \multirow[b]{2}{*}{ Trees absent } \\
\hline & & $\begin{array}{l}\text { Low rainfall (upto } \\
250 \mathrm{~mm} \text { ) }\end{array}$ & $\begin{array}{l}\text { High rainfall (upto } \\
250-350 \mathrm{~mm} \text { ) }\end{array}$ & \\
\hline Woody perennials & $\begin{array}{l}\text { Acacia senegal, } \\
\text { Prosopis cineraria, } \\
\text { Capparis decidua, } \\
\text { Ziziphus mummularia, } \\
\text { Calotropis procera, } \\
\text { Prosopis juliflora }\end{array}$ & $\begin{array}{l}\text { P. cineraria, Acacia } \\
\text { senegal, Calligonum } \\
\text { polygonoides, } \\
\text { Clerodendrum } \\
\text { phlomoides, Haloxyon } \\
\text { salicornicum, Aerva } \\
\text { pseudotomentosa } \\
\text { Calotropis procera, } \\
\text { Leptadenia } \\
\text { pyrotechnica }\end{array}$ & $\begin{array}{c}\text { Prosopis cineraria, } \\
\text { Tecomella undulata, } \\
\text { Balanites aegyptiaca } \\
\text { Acacia jacquemontii, } \\
\text { Calotropis } \\
\text { procera, Lycium } \\
\text { barbarum, Balanites } \\
\text { aegyptiaca, } \\
\text { Maytenus } \\
\text { emarginatus }\end{array}$ & $\begin{array}{c}\text { P. juliflora is invador Suaeda fruticosa, Salsola } \\
\text { baryosama, Haloxylon recurvum, Peganum harmala, } \\
\text { Trianthema portulacastrum }\end{array}$ \\
\hline $\begin{array}{l}\text { Herbaceous annuals } \\
\text { and perennials }\end{array}$ & $\begin{array}{l}\text { Aristida mutabilis, } \\
\text { Eragrostis spp. Cleome } \\
\text { papillosa, Boerhaavia } \\
\text { elegans }\end{array}$ & $\begin{array}{l}\text { Dipterygium glaucum, } \\
\text { Indigofera cordifolia, } \\
\text { Tephrosia tenuis, } \\
\text { Cenchrus prieurii, } \\
\text { Tribulus alatus, } \\
\text { Brachiaria ramosa }\end{array}$ & $\begin{array}{l}\text { Corchorus tridense, } \\
\text { Tribulus terrestris, } \\
\text { Heliotropium } \\
\text { strigosum, } \\
\text { Rhynchosia } \\
\text { minima, Brachiaria } \\
\text { reptens, Gisekia } \\
\text { pharnaceoides. }\end{array}$ & $\begin{array}{l}\text { Chloris virgata, Portulaca oleracea, Trianthema } \\
\text { portulacastrum, Echinochloa colonum }\end{array}$ \\
\hline
\end{tabular}

Each vegetation type has specific trees, shrubs, forbs, grasses and seasonals [7]. Though ephemeral/seasonals are specific to each vegetation type, their common occurrence within two or more vegetation types indicates that their niche requirements are met there. It is important to note that most of the ephemerals often form pure colonies under trees and shrubs. In fact, these are the pioneer species in succession of vegetation on these habitats when their dominance is $80-100 \%$ in the beginning of colonization and ecesis and it declines to often $20-30 \%$ in the sub-climax to climax formation.

\section{Life Cycle Of Ephemerals}

Besides showing spatial specificity, some ephemerals are unique in their temporal presence; being 'accidental vegetation [8]. Accidental vegetation appears only during high rainfall; say once in the years when rainwater collects in depression where these species come up. Monsoon herbage act as 'rain-gauges' i.e. these will germinate only after a particular amount of rain is received in one event. In contrast, there are species which germinate at the very first event of rainfall irrespective of amount. These include large number of species of Indigofera, Aristida and Cenchrus. If

\section{Composition of Herbage in Protected and Degraded Conditions}

Table 3: Botanical composition of herbage in protected $(\mathrm{P})$ and unprotected (Unp) grazinglands in three rain fall situations in Indian arid zone.

\begin{tabular}{|c|c|c|c|c|c|c|}
\hline \multirow[t]{3}{*}{ Species } & \multicolumn{6}{|c|}{ Site (Annual precipitation in $\mathbf{m m}$ ) } \\
\hline & \multicolumn{2}{|c|}{ Chandan $(150 \mathrm{~mm})$} & \multicolumn{2}{|c|}{ Beechwal $(250 \mathrm{~mm})$} & \multicolumn{2}{|c|}{ Palsana $(350 \mathrm{~mm})$} \\
\hline & $\mathbf{P}$ & Unp & $\mathbf{P}$ & Unp & $\mathbf{P}$ & Unp \\
\hline \multicolumn{7}{|l|}{ Grasses } \\
\hline 1. Aristida funiculata & + & + & + & + & + & + \\
\hline 2. Brachiaria ramosa & - & + & - & - & - & - \\
\hline 3. Cenchrus biflorus & + & - & + & + & - & + \\
\hline 4. Cenchrus prieuri & + & + & & & & \\
\hline
\end{tabular}

there is no successive rain with in a span of 15-20 days these will start flowering and set seeds as early as 20 days to 40-50 days. This has been confirmed by sequential sampling of permanent plots where seasonal variation in monsoonal vegetation has been charted and measured on rocky habitats [9], alluvial plains [10] and semi rocky habitats [11]. Their findings are: Within 21 days of rain, annuals were maximum 33.58\% in August and declined to $13.6 \%$ by end of September on rocky habitats of Kailana, near Jodhpur. Likewise, maximum dominance of annual grasses was $14 \%$ within 18 days of rain and it declined to almost $1 / 3$ i.e., $4.6 \%$ by the end of September on alluvial plains in Jodhpur. It has been found that at each germination event, $20-25 \%$ seeds of entire seed bank germinate and remaining are dormant. This is an ecological adaptation in annuals to germinate only a fraction of total seed bank so as to preserve the rest for next rain event. This ensures that if seedlings die or disappear without completing life cycle and formation of seeds, the remaining seed bank portion held with in soil is able to regenerate and continue the progeny. If however, there are continuously well distributed spells of rain, they continue to grow vegetatively till they face water stress. Growth up to 50-60 cm height has been observed in these species in case of prolonged wet spell. This indicates the plasticity in annual habit of these plants. 


\begin{tabular}{|c|c|c|c|c|c|c|}
\hline 5. Tragus biflorus (=roxburghii) & + & - & & & & \\
\hline 6. Eragrostis spp. & + & - & + & + & + & + \\
\hline 7. Latipes senegalensis & & & & + & & \\
\hline 8. Dactyloctenium aegyptium & & & & & - & + \\
\hline 9. Perotis indica & & & & & + & + \\
\hline 10. Fimbristylis spp. & & & & & + & - \\
\hline \multicolumn{7}{|l|}{ Forbs } \\
\hline 11. Polygala erioptera & & & + & + & & \\
\hline 12. Olden landia aspera & & & + & + & & \\
\hline 13. Mollugo nudicaulis & & & + & + & & \\
\hline 14. Cleome viscosa & - & - & + & + & & \\
\hline 15. Arnebia hispidissima & - & - & + & + & + & + \\
\hline 16. Anticharis linearis & - & - & + & - & & \\
\hline 17. Blepharis linearifolia & - & - & - & - & & \\
\hline 18. Corchorus tridens & + & - & + & - & & \\
\hline 19. Eurphorbia prostrate & + & - & + & - & & \\
\hline 20. Gisekia pharnaceoides & + & - & + & + & & \\
\hline 21. Heliotropium strigosum & + & - & + & + & & \\
\hline 22. Indigofera lin ifolia & + & - & + & + & + & - \\
\hline 23. Indigofera cordifolia & + & - & + & + & + & - \\
\hline 24. I. sessiliflora & + & - & & & & \\
\hline 25. Tribulus terrestris & + & - & & & + & - \\
\hline 26. T. alatus & - & + & & & & \\
\hline 27. Tephrosia tenuis & + & + & & & & \\
\hline 28. Euphorbia granulate & + & - & & & + & - \\
\hline 29. Farsetia hamiltonii & + & - & + & + & & \\
\hline 30. Indigofera linnaei & - & + & + & + & - & + \\
\hline 31. Portulaca sp. & - & - & + & + & . & \\
\hline 32. Solanum surratense & - & - & + & + & & \\
\hline 33. Sporobolus diander & & & & & + & - \\
\hline 34. Sesamum indicum & & & & & + & - \\
\hline 35. Borreria hispida & & & & & + & + \\
\hline 36. Ageratum conyzoides & & & & & + & - \\
\hline 37. Achyranthes aspera & & & & & + & - \\
\hline 38. Alysicarpus monilifer & & & & & + & - \\
\hline 39. Alysicarpus nudicaulis & & & & & + & + \\
\hline 40. Atylosia scarabaeoides & & & & & - & + \\
\hline 41. Commelina benghalensis & & & & & + & + \\
\hline 42. Euphorbia hirta & & & & & - & + \\
\hline 43. Heliotropium marifolium & & & & & - & + \\
\hline 44. Peristrophe bicalyculata & & & & & + & - \\
\hline 45. Polycarpea corymbosa & & & & & + & + \\
\hline 46. Rhyncosia minima & & & & & + & - \\
\hline 47. Indigofera hochstetteri & & & & & + & - \\
\hline 48. Justicia simplex & & & & & + & - \\
\hline 49. Kyllinge monocephala & & & & & - & + \\
\hline 50. Launea nudicaulis & & & & & + & - \\
\hline 51. Tridax procumbens & & & & & + & - \\
\hline
\end{tabular}


The composition of seasonals undergoes a drastic change upon grazing. A comparison of protected and grazed paddocks of grazingland at the end of 3 years and receiving $150 \mathrm{~mm}, 250 \mathrm{~mm}$ and $400 \mathrm{~mm}$ rain at Chandan, Beechwal and Palsana respectively revealed that grazed sites had preponderance of unpalatable annual forbs and such grasses which have awns that deter animals (Table 3) from grazing [12]. Analysis of dominance revealed that in low rainfall zones, seasonals have RIV of nearly 10 in protected conditions and this increased three times upon degradation. In $250 \mathrm{~mm}$ rainfall zone, the RIV of seasonals nearly 12 in protection became double i.e. 24.33 in degraded conditions. In $400 \mathrm{~mm}$ rainfall, the seasonals dominated by having RIV of 54 under protection and 75 under degradation. Thus dominance of monsoon seasonals increased with increasing annual rainfall and increased grazing stress.

\section{Production Potential}

There are a large number of studies on estimation of dry matter yield of rainy season ephemerals at regional, landscape and local level. Shankar and Kumar [13] estimated Aristida - Oropetium cover on 2526sq. km area in Jaisalmer with their yield as low as $5 \mathrm{~kg} / \mathrm{ha}$. However, contribution of seasonals varied from $2-27 \%$ in total dry matter that was $180 \mathrm{~kg}$ to $922 \mathrm{~kg} / \mathrm{ha}$ on different habitats in Jaisalmer. Potential of fair condition class grazingland was estimated as air dry $14 \mathrm{~kg} /$ ha for Aristida- Eragrostis type, 700 $\mathrm{kg} / \mathrm{ha}$ in Digitaria adscendens type, $3430 \mathrm{~kg} / \mathrm{ha}$ in Echinochloa colonum type and $4570 \mathrm{~kg} / \mathrm{ha}$ in Dachtyloctenium aegyptium in Jalore district [14]. At landscape level, production of palatable monsoonals in 3 landscapes in Churu district was 174, 250 and $567 \mathrm{~kg} /$ ha on forest floor, a planted grazingland and moderately degraded natural grazing land (Table 4). In Sikar, a protected site has $491 \mathrm{~kg} / \mathrm{ha}(92.9 \%)$ palatable and $40 \mathrm{~kg}$ (7.1\%) unpalatable herbage. The adjoining unprotected site had $230 \mathrm{~kg} /$ ha palatable (71\%) and $95 \mathrm{~kg} / \mathrm{ha}$ unpalatable herbage biomass [12]. Not only the total herbage yield but also yield of palatable species decline upon indiscriminate grazing. This also indicated a potential of regeneration of seasonal palatable herbage to the tune of nearly half a ton per hectare by mere protection.

Table 4: Production potential of grazingland herbage (kg.ha) in Churu District.

\begin{tabular}{|c|c|c|c|}
\hline Plant type & Forest floor at Taranagar & Protected grazing land at Changoi & Unprotected grazingland at Bighrain \\
\hline Perennial herbage & 568.151 & 281.489 & 140.097 \\
\hline Annual palatable herbage & 173.623 & 249.817 & 567.257 \\
\hline Annual unpalatable herbage & 23.101 & 129.100 & 60.357 \\
\hline
\end{tabular}

Table 5: Herbage Yield of different grass cover types on different habitats in Guhiya catchment [15].

\begin{tabular}{|c|c|c|c|c|}
\hline S.No. & Grass Cover type & Habitat & Average Drymatter yield (kg/ha) & Range (kg/ha) \\
\hline 1 & $\begin{array}{l}\text { Oropetium thomaeum (Linn.f.) trin., Eragrostis } \\
\text { ciliaris (Linn.) R. Br. }\end{array}$ & $\begin{array}{l}\text { Hill Older alluvial plain Buried } \\
\text { pediment }\end{array}$ & $\begin{array}{l}449 \\
242 \\
283\end{array}$ & $\begin{array}{l}270-645 \\
160-384 \\
224-342\end{array}$ \\
\hline 2 & $\begin{array}{l}\text { Dactyloctenium sindicum Boiss., } \\
\text { Eleusine compressa (Forsk.) } \\
\text { Aschers. Et Schweinf. }\end{array}$ & $\begin{array}{l}\text { older alluvial plain } \\
\text { Lower hills \& piedmonts } \\
\text { Younger alluvial plain }\end{array}$ & $\begin{array}{l}553 \\
1066 \\
464\end{array}$ & $\begin{array}{c}132-1098 \\
1066 \\
464\end{array}$ \\
\hline 3 & $\begin{array}{l}\text { Sporobolus marginatus Hochst., } \\
\text { Chloris virgata Sw. }\end{array}$ & $\begin{array}{l}\text { Saline older alluvial plain } \\
\text { Older alluvial plain }\end{array}$ & $\begin{array}{c}1045 \\
168\end{array}$ & $\begin{array}{c}828-1528 \\
168\end{array}$ \\
\hline 4 & $\begin{array}{l}\text { Cenchrus ciliaris Linn., } \\
\text { Cenchrus setigerus Vahl. }\end{array}$ & $\begin{array}{l}\text { Older alluvial plain } \\
\text { Obstruction dune }\end{array}$ & $\begin{array}{l}288 \\
700\end{array}$ & $\begin{array}{l}288 \\
700\end{array}$ \\
\hline 5 & $\begin{array}{l}\text { Dichanthium annulatum (Forsk.) Stapf. } \\
\text { Desmostachya bipinnata (Linn.) Stapf. } \\
\text { Cynodon dactylon (Linn.) Pers. }\end{array}$ & $\begin{array}{l}\text { Older alluvial plain } \\
\text { Saline older alluvial plain }\end{array}$ & $\begin{array}{r}404 \\
1488\end{array}$ & $\begin{array}{c}160-785 \\
1488\end{array}$ \\
\hline 6 & Aristida Spp. & $\begin{array}{c}\text { Piedmont } \\
\text { Older alluvial plain }\end{array}$ & $\begin{array}{l}470 \\
332\end{array}$ & $\begin{array}{c}380-645 \\
332\end{array}$ \\
\hline 7 & $\begin{array}{c}\text { Chrysopogon fulvus (Spreng.) } \\
\text { Chiov }\end{array}$ & Hills \& Piedmont & 1231 & $1048-1414$ \\
\hline
\end{tabular}


Its dependency on rain amount was proved by [15]. Estimates of grazable biomass in Guhiya catchment covering Pali and southem Jodhpur district (Table 5) revealed that predominantly seasonal grazinglands have approximate $449-470 \mathrm{~kg} / \mathrm{ha}$ dry matter yield, which is comparable to such grazing lands supporting perennials species. A scope of improvement in yield and carrying capacity was also predicted [16]. At village level, estimates in Bikaner district revealed that herbage yield in overgrazed native grazinglands was nearly half (330.98 kg/ha) of the nearby protected area $(610.65 \mathrm{~kg} /$ ha) which was fenced for two years (Table 6). It is interesting to note that even under protection the bulk of dry matter, i.e. nearly $44 \%$ was by a single monsoon legume i.e. Indigofera cordifola (Table 6) though there were other contributors like Cenchrus biflorous, Tribulus alatus, Farestia hamiltonii and Mollugo cerviana, their exact contribution was not reported by [17]. But this estimate amply proves that monsoon seasonals not only make up $34 \%$ of floristic composition, they also make up over $50 \%$ of biomass of grazing lands which is palatable too, in both protection and degraded conditions.

Table 6: Cover, dominance and herbage yield in a protected $(\mathrm{P})$ and unprotected (Unp) village grazinglands in six villages in Bikaner [17].

\begin{tabular}{|c|c|c|c|c|c|c|}
\hline \multirow[t]{2}{*}{ Species } & \multicolumn{2}{|c|}{ Cover $(\%)$} & \multicolumn{2}{|c|}{ Dominance (RIV) } & \multicolumn{2}{|c|}{$\begin{array}{c}\text { Herbage yield }(\mathrm{kg} / \mathrm{ha})(\% \\
\text { contribution of yield in brackets) }\end{array}$} \\
\hline & $\mathbf{P}$ & Unp & $\mathbf{P}$ & Unp & $\mathbf{P}$ & Unp \\
\hline \multicolumn{7}{|c|}{ Grasses } \\
\hline Lasiurus sindicus & 2.33 & 0.23 & 16.71 & 4.02 & \multirow{4}{*}{$\begin{array}{c}257.32 \\
(42.15)\end{array}$} & \multirow{4}{*}{$\begin{array}{c}81.33 \\
(24.49)\end{array}$} \\
\hline Dactyloctenium sindicum & 0.2 & 0.2 & 7.94 & 8.65 & & \\
\hline Cenchrus biflorus & 0.5 & 0.067 & 4.86 & 2.31 & & \\
\hline Eleusine compressa & 0.03 & 0.817 & 24.69 & 27.5 & & \\
\hline \multicolumn{7}{|c|}{ Legumes } \\
\hline Indigofera cordifolia & 0.3 & 0.3 & 7.48 & 8.59 & $\begin{array}{l}268.00 \\
(43.88)\end{array}$ & $\begin{array}{l}21.33 \\
(6.42)\end{array}$ \\
\hline \multicolumn{7}{|c|}{ Others } \\
\hline Convovulus microphyllus & 0.417 & 1.01 & 13.78 & 21.4 & \multirow{6}{*}{$\begin{array}{c}85.33 \\
(13.97)\end{array}$} & \multirow{6}{*}{$\begin{array}{l}229.32 \\
(69.09)\end{array}$} \\
\hline Cyperus bulbosus & 0.08 & 0.08 & 4.74 & 5.05 & & \\
\hline Tribulus alatus & 0.13 & 0.187 & 6.73 & 8.11 & & \\
\hline Farsetia hamiltonii & 0.21 & 0.217 & 6.88 & 7.67 & & \\
\hline Heliotropium strigosum & 0.13 & 0.133 & 4.51 & 5.0 & & \\
\hline Mollugo cerviana & 0.006 & 0.007 & 1.66 & 1.70 & & \\
\hline \multicolumn{5}{|c|}{ Total } & 610.65 & 331.98 \\
\hline
\end{tabular}

Similar conclusion was reached by [18] in seven-year monitoring of rangelands of Lawan in Jaisalmer stating that "--- major contribution in all grazing plots was from Indigofera cordifolia (1.26-1.98\%) and less than one percent form perennial grasses". In extremely degraded conditions however, all palatable are removed and unpalatable biomass also remain $1 / 2$ and $1 / 3$ of that in protected situations. The important implication of this finding is that nearly $70 \%$ of grazingland in Indian arid zone are degraded while $14 \%$ are in fair, $13 \%$ in good and $2-3 \%$ have excellent condition class [7]. It is these $70 \%$ areas which have poor perennial plant cover but a preponderance of monsoon seasonals and these ephemerals constitute available grazable material @ $500 \mathrm{Kg} /$ ha to sustain liverstock.

\section{Ephemeral's Response to Grazing}

Impact of different levels of grazing on cover of selected annual forbs and annual grasses in permanent quadrats and transects in arid and semi arid regions was investigated in one monsoon season at two sites in India: low rainfall arid site $(240 \mathrm{~mm} / \mathrm{year})$ at Chandan experimental site of CAZRI in Jaisalmer district in western India and a high rainfall semi arid site $(400 \mathrm{~mm} / \mathrm{year})$ at Pali experimental site of CAZRI in Pali district [19]. Treatments were: No grazing (control), $50 \%$ or half of carrying capacity (3 sheeps/ha), $100 \%$ of carrying capacity (6 sheeps /ha) and $50 \%$ more than carrying capacity ( 8 sheeps/ha). Mild grazing of 3 sheep/ ha and sometimes even 6 sheep/ha at arid Chandan site enhanced the cover of three annual grasses, Aristida mutabilis, C. biflorus, Latipes senegalensis from July to October. Such an increase was more than that in control or 8 sheep/ha treatment, confirming the fact that mild grazing promotes the growth. In contrast, annual forbs (Indigofera cordifolia, I. linifolia, I. hochstetteri and Gisekia pharnaceoides) cover declined with increasing grazing pressure. Response to increasing grazing pressure was different at semiarid Pali site: though Aristida mutabilis increased with increasing 
grazing pressure, the other forbs species also increased up to September and declined later, with increasing grazing pressure.

This was also confirmed by positive significant correlation between cover and grazing intensity i.e., increases in cover with increasing grazing pressure in Aristida mutabilis. Importantly, correlation coefficient between grazing pressure and cover of Indigofera hochstetteri, I. cordifolia, I. linifolia at arid site and C. pumila at semi arid site was strongly negative. Thus same species behaves differently in two different rainfall situations. It emerged from above that annual species after being nibbled or partly grazed by sheep re-sprout and assume growth in semi arid situation, behaving much as multi cut fodder corp. These findings were in conformity with results of an earlier grazing experiment of three years at Kailana, Jodhpur where per cent plant cover increased from 4.8 to $7.42 \%$ and forage yield, 28.3 to $29.8 \mathrm{~kg} / \mathrm{ha}$ after grazing [20].

In the same experimental paddocks at Chandan in Jaisalmer district of western India, phenological changes at monthly intervals were also recorded under different grazing pressures. Concurrently, palatability of species as and when the sheep bites was also noted for one hour each during morning (8-10AM), noon (12-2PM) and evening (4-8PM). Results revealed that vegetative phase was shortened and flowering, fruiting and seed set occurred earlier in perennial like Lasiurus sindicus as the grazing pressure increased [21]. Thus compressing the vegetative phase emerged as a mechanism of evading grazing pressure in this perennial grass. Reverse was noted in all the ephemeral species. Vegetative phase was prolonged with increasing intensity of grazing and thus seed setting was delayed in ephemeral species Aristida funiculata, Indigofera cordifolia, Indigofera linifolia and Corchorus tridense. This shift in phenophase could be related to their relative palatability. Since annual species germinate and grow with their fresh foliage during the monsoon rains, annuals (due to fresh foliage) were eaten in preference over the perennials. This preferred removal of annuals due to their palatability, if continued unabated, would finally remove them completely due to overgrazing. In fact, such a shift in phenophases, induced by increase in grazing intensity, can effectively be used as indicator of beginning of deterioration of rangeland health. And this becomes the start point of desertification in grazing lands in deserts. Moderate grazing is therefore, desirable for maintaining range health for sustainable grazing. On the other hand, it is also important to realize this potential of monsoon ephemerals as grazing material as by way of corollary these can be sown and then cut, not grazed, and fed to animals.

In order to further understand the role of herbaceous annual and seasonal vegetation, another grazing study was undertaken for two consecutive years in Lasiurus sindicus dominated protected grazingland at experimental area at Chandan in Jaisalmer district by Kumar et al. [22]. There were five grazing treatments:T-1: Control
(No grazing); T-2: Optimum carrying capacity with supplemental feed (6 sheep grazing); T-3: Optimum carrying capacity without supplemental feed (6 sheep grazing); T-4: Double the carrying capacity with supplemental feed (12 sheep grazing) and T-5: Double the carrying capacity without supplemental feed (12 sheep grazing). Results revealed that irrespective of supplemental feed, $70-80 \%$ of L. sindicus cover declined in paddock with double the carrying capacity (T-4 and T-5). This study again proved findings in previous para i.e., preferential consumption of seasonal and low perennials such as Ochthochloa compressa and annual Cenchrus biflorus in monsoon and post-monsoon. Grazing animals did not eat perennial species as if these have been left by them for future consumption when these ephemerals dry, die and no more available say, after December. This postponement in consumption of perennials effectively prolonged the duration of range-use. On the other hand, as the grazing pressure increased, biomass declined by $80 \%$ in two years i.e., from 461.5 (T-2), 306.6 (T-3), 450.5 (T-4) and 341,1 (T-5) kgha-1 to 70.3 (T-2), 29.6 (T-3), 28.7 (T-4) and 15.4 (T-5) kgha-1. In rain driven ecosystem of arid lands, even a small variation in the quantum, spread and timing of rainfall causes major effect on vegetation composition [23]. These have cascading effects making the whole system vulnerable to drought and adversely affecting sustainable productivity of the rangeland ecosystem [24]. This study thus, concluded that rainy season ephemerals by way of their preferential consumption give temporary or seasonal rest to perennials like L. sindicus enabling it to grow and recover. Vetter et al. [25] while discussing such differences in composition, structure, diversity and forage production potential of vegetation under different grazing intensities reasoned that these ephemerals can draw water from whole soil profile throughout the growing seasons where as climax grasses withdraw water from deeper layers of 2-5m during droughts [23].

Thus, resource utilization is partitioned to be optimally used among ephemerals and perennials resulting in better growth of both these components. Consequently, grazable material becomes available from the same grazing land for a longer duration and that imparts resilience to the grazing land system. Fynn [26] also reported that short, nutritious grasses in functional wet seasons habitats facilitate optimum intake of nutrients and energy for lactating females, for optimal calf growth and building body stores. Heterogeneity in vegetation composition due to ephemerals was also emphasized for achieving optimum grazing use by [28]. Evidently, Kumar et al. [22] in their study also related the spatial patterns created by patches of seasonal vegetation in the landscape and temporal patterns of biomass (= productivity) availability of seasonals in post monsoon and perennials in winter and summer with long term sustainability. Fynn [26] also concluded that grazing based on spatial and temporal variability in forage quality and quantity would be more sustainable. Nutritionally also, seasonal vegetation having higher crude protein than perennial grasses 
[28] meets the nutritional needs of the livestock. Utilizing above mentioned spatial and temporal patterns was also recommended as best range utilization strategy by [27]. But Kumar et al. [23] found that free range animals in monsoonal rangelands themselves do selective grazing based on seasonal availability of biomass i.e., they graze annuals first (August to November) and then perennials (December to April) and both litter and perennials in summer. A mix of cattle, goat and sheep would further optimize to increase range utilization.

Ephemerals/seasonals, therefore, not only provide heterogeneity and complexity to the grazing landscape, they also complement the nutritional needs as well as prolong the period of range use thereby delaying the onset of degradation. This spatial heterogeneity imparted by seasonal vegetation in an overall matrix of perennial tall grasses and woody perennials need to be managed optimally by grazing management of both seasonals and perennials. It was therefore, concluded ".... that

a) arid rangelands have intrinsic heterogeneity in species composition,

b) this mix of seasonal and perennials (= heterogeneity) is ably supported by the landscape by way of partitioning of resources,

c) lifecycle patterns of seasonal fits well to meet the nutritional needs of livestock and

\section{Ephemerals as Crop Area Weeds}

Table 7: Dry matter yield of different crops and their weeds in Guhiya catahment. (Source: Shankar and Kumar, 1984).

\begin{tabular}{|c|c|c|c|c|c|c|c|c|c|}
\hline \multirow[t]{2}{*}{ Habitat } & \multirow[t]{2}{*}{ Soil texture } & \multirow[t]{2}{*}{ Crop } & \multicolumn{2}{|c|}{ Dry matter kg/ha } & \multirow{2}{*}{$\begin{array}{l}\text { Weed/crop } \\
\text { ratio in yield }\end{array}$} & \multicolumn{4}{|c|}{ Botanical composition \% } \\
\hline & & & Crop & Weeds & & Crops & Weeds grasses & Legumes & Others \\
\hline \multirow[t]{4}{*}{$\begin{array}{l}\text { Older alluvial } \\
\text { plain }\end{array}$} & Sandy loam & Green gram & 158.5 & 740.5 & 4.67 & 17.63 & 51.1 & 10.46 & 20.85 \\
\hline & Clayey & Pearl Millet & 293.0 & 1289.0 & 4.39 & 18.52 & 1.71 & - & 79.77 \\
\hline & Clay Loam & Pearl Millet sorghum & 414.0 & 1195.0 & 2.88 & 25.73 & 36.1 & 8.08 & 30.08 \\
\hline & Clayey & Mehendi & 6.0 & 271.2 & 45.20 & 2.53 & 5.06 & & 92.41 \\
\hline \multirow[t]{7}{*}{$\begin{array}{l}\text { Pediment Older } \\
\text { alluvial plain }\end{array}$} & Clay loam & $\begin{array}{l}\text { Pearl millet \& } \\
\text { Gingelly }\end{array}$ & 503.0 & 1564.0 & 3.10 & 24.33 & 21.5 & 10.11 & 44.08 \\
\hline & Sandy loam & Moth been & 859.0 & 1131.0 & 1.32 & 85.90 & 1.05 & - & 12.05 \\
\hline & Clay Loam & Sorghum & 1513.0 & 589.0 & 0.39 & 71.98 & 0.19 & 15.15 & 12.28 \\
\hline & Sandy loam & Sorghum & 1898.0 & 691.0 & 0.364 & 56.51 & 11.69 & 11.98 & 20.21 \\
\hline & Sandy loam & $\begin{array}{l}\text { Mothbeen, Green } \\
\text { gram \& Gingelly }\end{array}$ & 1508.0 & 489.0 & 0.32 & 75.49 & 6.03 & 0.98 & 17.49 \\
\hline & Sandy loam & $\begin{array}{c}\text { Greengram \& Cluster } \\
\text { bean }\end{array}$ & 259.0 & 970.0 & 3.74 & 21.07 & 35.47 & 0.65 & 42.79 \\
\hline & Sandy loam & Pearl millet & 1196.0 & 655.7 & 0.54 & 64.60 & 22.54 & - & 12.86 \\
\hline
\end{tabular}

Ephemerals appear to be out of place in crop lands and hence called weeds. Desert farmers know the utility of these seasonals as valuable forage. Quite often, especially the monsoon ephemerals are collected, air dried and stored in livestock yard for future used. Heaps of such air dried palatable ephemerals are stacked species wise as an effective strategy of escaping drought by way d) grazing of such seasonal vegetation thus helps sustain perennial tussocky rangelands for longer range-use ...."

Such a functional role of ephemerals as revealed in the aforementioned studies in respect of grazing animals has also been found in an entirely different context by Bhardwaj et al. [29] who reported the importance of ephemerals in enhancing the population of critically endangered bird, the Great Indian Bustard Ardeotis nigriceps with a remaining population of $\sim 200$ birds (IUCN 2011). Biased habitat preference by Great Indian Bustard for new closures which were ploughed instead of old ones prompted a detailed vegetation analysis by [30] amongst different landscape units. The newly ploughed sited had regeneration of lot of ephemerals in the ensuing rains compared to the parched sites in the old ones. This led to devise a very simple management strategy of ploughing the open areas in mosaic pattern for breaking the crust laden hard surface without disturbing the Lasiurus sindicus and other perennial grasses, shrubs and trees of old closures annually just before onset of rains. Selective ploughing brought out the stored seed bank of annuals on to the surface enabling them to use moisture of the very first rain and germinate to grow further in subsequent rains. In fact, such slight amount of disturbance is known to increase diversity in all dry land ecosystems and is a desirable management intervention for the conservation of critically endangered Great Indian Bustard. of providing feed to livestock during ensuing drought when crops fail. It becomes therefore, imperative to assess the quantity of such ephemeral forage in crop fields. A study to assess their biomass revealed that weed biomass often far exceeded their companion crop [30] i.e., their biomass was 2 to 4 times more than crop (Table 7). These are mostly palatable weeds giving 655 to $1564 \mathrm{~kg} /$ 
ha of dry matter depending upon preponderance of weeds. Thus seasonal ephemerals as weed contribute immensely to livestock feed but estimates are not available to assess their contribution at landscape level.

\section{Role of Ephemerals in Rehabilitation of Mine Spoils}

Though perennials are preferred for rehabilitation of degraded landslike mine spoils, butonce the seeds bankis added to soil, annuals by virtue of their repid turn over, play a key role in accelerating process of rehabilitation. Kumar et al. [31] reported as many as 14 self regenerated annuals, equivalent to that of perennials in freshly rehabilitated plots of gypsum mine spoil in Barmer, an extreme arid district in western Rajasthan. These provided organic matter to soil facilitating growth of shrubs and trees. These can be therefore, easily employed to grow and prepare niche for future generations of plants. In another rehabilitation programme for backfilled areas after lignite mining in Barmer (1999-2004), surface layering of local soil and murram was carried out and then planted with eight different tree species, seven shrub species and one perennial grass [32]. Monitoring the self- regenerated natural vegetation in these treatment blocks revealed that irrespective of treatments, all blocks had immense regeneration of ephemerals that constituted $46-48 \%$ of total self regenerated species that varied from 39 to 75 species in five rehabilitated blocks. Appearance and growth of nearly half of regenerated vegetation being ephemeral proved their crucial role in accelerating the process of rehabilitation by way of organic matter build up and its rapid turn over that later on supports growth of other companion planted species. Evidently, understanding the role of ephemerals as organic matter builder, succession facilitator and ecological moderator deserve deeper studies so as to optimize cost of such programmes and achieve faster system recovery in reclamation of degraded and drastically disturbed lands, especially in arid regions.

\section{Ephemerals as Medicinal Plants}

A survey of ethno medicinal plants in 128 villages in four arid districts in western India namely Jaisalmer, Barmer, Bikaner and Jodhpur from year 2001 to 2005 revealed 131 taxa of medicinal importance (Kumar and Parveen, 2004) [33]. Of these, herbs were maximum i.e. 52 (39.69\%) followed by 29 shrubs (22.13\%), 25 trees (19.84\%), 11 climbers (8.39\%), 9 grasses (6.87\%), 3 sedges (2.29\%) and 1 fungus $(0.67 \%)$. Of the total species, 70 were perennials (53.43\%) and 61, ephemeral or annuals including crops (46.57\%). These values broadly match the Raunkiarian's life forms in this desert in which nearly $49 \%$ are therophytes or annuals and $51 \%$ are other life forms which are perennials Mertia and Bhandari [34]. The 29 ephemeral medicinal species reported from wild grazing lands in this study are :Abutilon indicum, Achyranthus aspera, Amaranthus viridis, Argemone mexicana, Blepharis sindica, Cenchrus biflorus ,Cleome viscosa, Corchorus depressus, Corchorus tridens, Cucumis callosus, Dicoma tomentosa, Digera muricata, Eclipta alba, Eragrostis minor, Euphorbia granulata, Fagonia indica, Heliotropium marifolium, Indigofera cordifolia, Mollugo cerviana, Mukia maderaspatana, Neurada procumbens, Pedalium murex, Phyllanthus fraternus, Polygonum plebieum, Portulaca oleracea, Pulicaria crispa, Sisymbrium irio, Solanum surattense and Tribulus terrestris. Almost all of these species come up naturally during monsoonal rains and hence are collected from wild and marketed. Many of these are immensely useful and are species of trade both nationally and internationally i.e., Tribulus terrestris, Pedalium murex, Solanum surratense, Fagonia indica, Blepharis linearifolia, Phyllanthus fraternus, and Eclipta alba. Thus these nature's herbals enhance the cash flow in the hand of farmers. But in view of their increasing demand, a need is felt to domesticate and bring them into cultivation so as to save their native gene pools in the wild from being lost. This will also diversify the cropping system and add to the sustainable livelihood of desert dwellers.

\section{Epilogue}

Ephemeral/seasonal species are important herbage component of natural grazing lands, pasture and croplands. They are the only plants available even after the perennials are removed. Persistence to withstand pressures and re-appear once the favorable situations occur, make them highly resilient component of desert vegetation. Their life cycle strategy is also more suited to adverse temporal sequential stresses caused by climatic aberrations. Plasticity in phenological stages exhibited by ephemerals during variable grazing treatments not only provides adaptive edge to survive in harsh desertic conditions but also imparts sustenance to the whole grazing land ecosystem. By way of providing heterogeneity in species composition, ephemerals prolong the duration of grazing land use and also complement the nutritional needs of livestock. This aspect has helped to design conservation strategy of critically endangered Great Indian Bustard in its native landscape by way of provisioning of foraging material by just enabling buried ephemerals seeds to come up on surface through ploughing and then germinating during rains.

They contribute up to $500-600 \mathrm{~kg} / \mathrm{ha}$ dry matter without any management in natural conditions. Grown properly under managed conditions, their yield can be enhanced many folds. This can be achieved by providing moisture just before flowering so that vegetative phase is prolonged, thus capitalizing its ecological properties to further increase its yield to double or treble. Experiments are however needed to confirm whether these could be used as multicut fodder crop in sole or as intercrop. In view of rich ephemeral flora in the Indian desert, we are advantageously placed to select a few chosen species for testing their potential as future livestock feed either raw or as feed cake. Not merely a source of livestock feed, these grazing lands are also cradle of as many as 61 herbal ephemerals which are being used by people as traditional 
medicine; many of them having immense trade potential that can add to the kitty of local farmers. In the ecological management of mine spoiled lands, ephemerals as an adjunct to perennial species have proved their potential as a cheaper and faster rehabilitation material.

\section{Acknowledgement}

I express my gratitude to Director, CAZRI, Jodhpur for providing facilities to carry out the work reviewed in this paper.

\section{References}

1. Kar A (2014) Agricultural land use in arid Western Rajasthan: Resource exploitation and emerging issues. Agropedology 24(2): 179-196.

2. Ramana Rao BV, Rao GGSN, Gupta BS, Malakar AR (1983) Influence of commencement of sowing rains, or crop production in dry districts of Rajasthan Mausam 34: 335-338.

3. Dabadghao PM, Shankarnayrayan KA (1973) The Grass covers of India. ICAR, New Delhi, India, pp. 718.

4. Singh Panjab (1988) Indian rangelands status and improvement Plenary Address to the 3rd International Rangeland Congress, New Delhi, India, pp. 40.

5. Bhandari MM (1990) Flora of Indian Desert. MPS Reports Jodhpur, India, pp. 435.

6. Satyanarayan Y (1964) Habitat and plant communities of the Indian Desert. in Proc. of Symp. on Problems of Indian Arid Zone. Min. of Education, Govt. of India, new Delhi, India, pp. 59-68.

7. Shankar V, Kumar S, Tyagi AK (1988) Grazing resources of arid and semi-aird ragions. In; Pastures and Forage Crops Research- A State of knowledge Report. 3rd International Rangeland Congress, new Delhi, India, pp. 63-85.

8. Kassas M (1966) Plant life in the desert. In E. Hills (Eds.) Arid Lands, Metheum, London: pp. 145-180.

9. Gaur YD, Satayanarayan Y (1967) Phytosociological Stuides of the Monsoon Vegetation of Rocky Habitat. Indian Forester 93(12): 806-814.

10. Satayanarayan Y, Gaur YD (1967) Phytosociological Variations in Floristic Composition of the Vegetation in the Arid Zone. 1. Monsoonal Vegetation of the Alluvial Plains. Annals of Arid Zone 6(2): 180-199.

11. Satynarayan Y, Gaur YD (1968) Phyotosociological Changes of Monsoon vegetation in Semi-Rocky Habitats. The Journal of the Indian Botanical Society XL VII (3-4): 371-381.

12. Anonymous (1978) Productivity of Arid Grazingland Ecosystem. Annual Progress Report of MAB Project, CAZRI pp. 91.

13. Shankar V, Kumar S (1987) Herbage yield and carrying capacity of grasslands in Jaisalmer district Trop. Ecol 28: 239-245.

14. Gupta RK, Saxena SK (1966) Habitat, Grassland Types and Forage Potential of Jalore District in Western Rajasthan. Annals of Arid Zone 5(2): 190-203.

15. Harsh LN, Sen DN (1977) Ecoclimate and the primary production of the Ephemerals in The Desert Ecosystem. Geobios 4: 190-193.

16. Kumar S, Shankar V (1986) Grass covers of the Guhiya catchment of the Upper Luni Basni, 1. Present and potential herbage production and carrying capacity. Indian Jouranl of Forestry 9(2): 126-131.

17. Kumar S (1991) Assessment of grazing resources and their demand availability status through rein use efficiency analysis in arid land villages- Forage Research 17(1): 45-54

18. Sharma SK, Ahuja LD, Yadav RPS, Verma CM (1980) Changes in the Botanical Composition in the Long Term Seasonal Grazing Paddocks of a Desert Rangeland Forage Research 6(1): 7-14.

19. Narita K, Mertia RS, Kumar S, Ichikawa S, Furukawa A (1997) Effects of different grazing intensities of sheep on arid and semi-arid grasslands in the Thar Desert, India, Tropics 7(1/2) : 105-114.

20. Das RB, Bhimaya CP (1971) Ecology of grassland of Western Rajasthan. Proceeding of Symposium on Problem of Indian Arid Zone 5(2): 222226.

21. Kumar S, Mertia RS, Narita K, Ichikawa S, Furukawa A (1999) Seasonal shits in phenology and palatability under various grazing pressures: Implication for rangeland health. In Eldridge D and Freudenberager D. People and Rangelands Building the Future. Proc. of VI International Rangeland congress: pp. 550-551.

22. Kumar S, JP Singh, K Venkatesan, BK Mathur, RK Bhatt (2017) Changes in seasonal vegetation and sustenance of tussocky arid rangelands under different grazing pressures. Range Management and Agroforestry 38(1): 35-42.

23. Snyman HA (1998) Dynamics and sustainable utilization of rangeland ecosystems in arid and semi-arid climates of southern Africa. Journal of Arid Environment 39(4): 645-666.

24. Yunlong C, B Smit (1994) Sustainability in Agriculture: a general review. Agriculture, Ecosystem and Environment 49(3): 299-307.

25. Vetter S, WM Goqwana, WJ Bond, W Trollope (2006) Effects of land tenure, geology and topography on vegetation and soils of two grasslands types in south Africa. African Journal of Range Forage Science 23(1): 13-27.

26. Fynn RWS (2012) Functional resource heterogeneity increases livestock and rangeland productivity. Rangeland Ecology and Management 65(4): 319-329.

27. McGranahan DA, KP Kirkman (2013) Multi-functional rangelands in southern Africa: Managing for production, conservation and resilience with fire and grazing. Land 2(2): 176-193.

28. Singh JP, BC Mondal, ML Soni, RK Beniwal (2006) Annual weeds add to the nutritional level of forage from desert rangelands. Annals of Arid Zone 45(2): 189-193.

29. Bhardwaj GS, KR Anoop, Prerna Sharma, Suresh Kumar (2017) Role of Herbaceous Vegetation in Habitat Utilization by Critically Endangered Great Indian Bustard Ardeotis Nigriceps (Vigors) in the Indian Thar Desert. Indian Forester 143(10): 975-984.

30. Shankar V, Kumar S (1984) Ecology of crop area weeds in the Guhiya catchment of the Upper Luni Basni in Rajasthan. J Econ Tax Bot 5(2): 321-327.

31. Kumar S, Sharma KD, Sharma BK, Gong LP (1988) Richness, diversity and evenness of vegetation upon rehabilitation of gypsum mine spoiled lands in the Indian Arid Zone Annals of Arid Zone 37(2): 139-145.

32. Kumar S, Praveen Kumar (2014) Rehabilitation of Lignite Mine Spoils in the Indian Arid Zone-An Ecological Approach. Annals of Arid Zone 53(3\&4): 121-136.

33. Kumar S and Farzana Parveen (2004). Traditional Ethnomedicinal Plants in the Indian Thar: Their Status in Natural and Possibilities of their Cultivation and Trading. CAZRI, Jodhpur. 216p

34. Mertia RS, Bhandari MM (1980) Vegetational adaptation in the extremely arid regions of the Indian Desert. In Mann HS (Eds.) Arid Zone Research and Development. Scientific Publishers, Jodhpur, India, pp. 155-164. 
(c) C) This work is licensed under Creative

To Submit Your Article Click Here:

Submit Article

DOI: 10.32474/CIACR.2018.04.000190

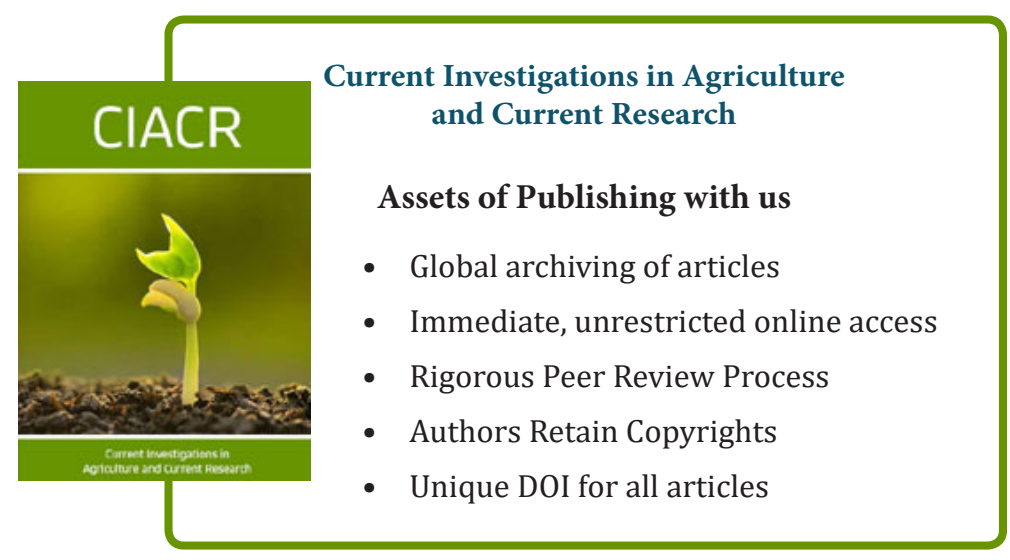

